\title{
An Elementary Theorem in Geometric Invariant Theory
}

\section{Citation}

Mumford, David. 1961. An elementary theorem in geometric invariant theory. Bulletin of the American Mathematical Society 67(5): 483-487.

\section{Published Version}

doi:10.1090/S0002-9904-1961-10647-2

\section{Permanent link}

http://nrs.harvard.edu/urn-3:HUL.InstRepos:9888892

\section{Terms of Use}

This article was downloaded from Harvard University's DASH repository, and is made available under the terms and conditions applicable to Other Posted Material, as set forth at http:// nrs.harvard.edu/urn-3:HUL.InstRepos:dash.current.terms-of-use\#LAA

\section{Share Your Story}

The Harvard community has made this article openly available.

Please share how this access benefits you. Submit a story.

Accessibility 


\title{
AN ELEMENTARY THEOREM IN GEOMETRIC INVARIANT THEORY
}

\author{
BY DAVID MUMFORD
}

Communicated by Raoul Bott, May 18, 1961

The purpose of this note is to prove the key theorem in a construction of the arithmetic scheme of moduli $M$ of curves of any genus. This construction, which relies heavily on Grothendieck's whole theory of schemes, may be briefly outlined as follows: first one defines the family $K$ of tri-canonical models of curves $C$ of genus $g$, any characteristic, in $\mathbf{P}^{5 g-6}$, as a sub-scheme of one of Grothendieck's Hilbert schemes [3]. Second, one maps $K \rightarrow A$, where $A$ (a sub-scheme of another Hilbert scheme) parametrizes the full projective family of the polarized Jacobians $J \subset \mathrm{P}^{N}$ of these curves. It may then be shown that $M$ should be the orbit space $K / \mathrm{PGL}(5 g-6)$; and it can also be shown that this orbit space exists if the orbit space $A / \operatorname{PGL}(N)$ exists. But, in general, given any family $A$ of polarized abelian varieties $V \subset \mathbf{P}^{N}$ invariant under projective transformations, $A / \mathrm{PGL}(N)$ does exist; for simplicity assume that a section serving as an identity is rationally defined in the whole family $A$. Then $A$ may be identified with the family of 0 -cycles $\mathfrak{A} \subset \mathbf{P}^{N}$ which are the points of order $m$ (suitable $m$ ) on the abelian varieties $V$. Now neglecting for simplicity the group of permutations of the $m^{2 \sigma}$ points of these 0 -cycles, this reduces the problem to constructing the orbit space $\left(\mathrm{P}^{N}\right)^{m^{20}} / \operatorname{PGL}(N)$. In this paper, an apparently very natural open sub-scheme ${ }^{1}\left(\mathbf{P}^{n}\right)_{0}^{m}$ $\subset\left(\mathbf{P}^{n}\right)^{m}$, any $n, m$, is constructed such that in fact $\left(\mathbf{P}^{n}\right)_{0}^{m}$ is a principal fibre bundle over its quotient by $\operatorname{PGL}(n)$. This result is apparently new even over the complex numbers. The methods used are entirely elementary, and no special techniques are used to deal with the generalization from varieties to schemes. Moreover, except for the replacement of $\mathbf{Z}$ by $k$, no changes are necessary or appear possible should the reader wish to consider the objects as varieties rather than schemes.

1. Let $\mathbf{P}^{n}$ be projective $n$-space over $\mathbf{Z},\left(\mathbf{P}^{n}\right)^{m}$ the $m$-fold product with itself. Let homogeneous coordinates in the $i$ th factor be $X_{0}^{(i)}$, $X_{1}^{(i)}, \cdots, X_{n}^{(i)}$, and for all $(n+1)$-tuples $i_{0}, i_{1}, \cdots, i_{n}$ let

$$
D_{i_{0}, i_{1}, \cdots, i_{n}}=\underset{0 \leqq k, l \leqq n}{\operatorname{Det}}\left(X_{l}^{\left(i_{k}\right)}\right) .
$$

\footnotetext{
${ }^{1}$ See also [2] for a slightly larger open sub-scheme.
} 
Define $\left(\mathbf{P}^{n}\right)_{0}^{m} \subset\left(\mathbf{P}^{n}\right)^{m}$ to be the "set of $m$-tuples" $\left(P_{1}, P_{2}, \cdots, P_{m}\right)$, $P_{i} \in \mathbf{P}^{n}$, such that less than $m /(n+1) P_{i}$ are contained in any single hyperplane of $\mathbf{P}^{n}$. This is immediately seen to be an open sub-scheme.

Proposition. Let $P=\left(P_{1}, P_{2}, \cdots, P_{m}\right)$ be a geometric point in $\left(\mathbf{P}^{n}\right)_{0}^{m}$.

(a) There are positive integers $N$ and $N_{0}$ such that for every $i, a$ monomial $\Pi^{(i)}$ in the $D^{\prime}$ s exists such that $\Pi^{(i)}(P) \neq 0$, and the degree of $\Pi^{(i)}$ in $X_{*}^{(j)}$ is $N$ if $j \neq i$, and $N-N_{0}$ if $j=i$.

(b) There is a positive integer $N$ such that for every $i \neq j$, a monomial $\Pi^{(i, j)}$ exists such that $\Pi^{(i, j)}(P) \neq 0$, and the degree of $\Pi^{(i, j)}$ in $X_{*}^{(\mathbf{k})}$ is $N$ if $k \neq i, j$, and $N+1$ if $k=i$, and $N-1$ if $k=j$.

Proof. Let $E$ be the real vector space of dimension $m$, and let $H$ be the convex cone spanned by the points

$$
P_{i_{0}, i_{1}}, \cdots, i_{n}=\left(x_{1}, x_{2}, \cdots, x_{m}\right),
$$

where $x_{i}=0$ if $i \neq i_{k}$, any $k, x_{i}=1$ if $i=i_{k}$, some $k, D_{i_{0}, i_{1}, \ldots, i_{n}}(P) \neq 0$. By means of assigning to each monomial in the $D$ 's the corresponding additive expression in the $P$ 's, the result (a) can readily be translated to the assertion that $(1,1, \cdots, 1) \in \operatorname{Int}(H)$. But if this is false, there is a linear functional on $E$, zero at $(1,1, \cdots, 1)$ and negative on $H$, i.e. there exist $\alpha_{1}, \cdots, \alpha_{m}$ such that

$$
\begin{aligned}
\sum_{k=0}^{n} \alpha_{i_{k}} \leqq 0 & \text { if } D_{i_{0}, i_{1}}, \cdots, i_{n} \\
\sum_{i=1}^{m} \alpha_{i} & =0 .
\end{aligned}
$$

Say without loss of generality that $\alpha_{1} \geqq \alpha_{2} \geqq \cdots \geqq \alpha_{m}$. Pick the sequence $i_{0}, i_{1}, \cdots, i_{n}$ as follows: $i_{0}=1 ; i_{1}=$ smallest $i$ such that $P_{1} \neq P_{i} ; i_{2}=$ smallest $i$ such that $P_{1}, P_{i_{1}}, P_{i}$ do not lie on a line; etc. It is easy to see that the hypothesis on $P$ implies $i_{n}-1<m /(n+1)$, hence $i_{n} \leqq[m /(n+1)]+1=\mu+1$, for instance. Now

$$
\begin{aligned}
0 & \geqq \mu \sum_{k=0}^{n} \alpha_{i_{k}} \\
& \geqq \mu \alpha_{1}+\mu n \alpha_{i_{n}} \\
& \left.\geqq \mu \alpha_{1}+\mu n \alpha_{\mu+1} \quad \quad \text { (since } \alpha_{\mu+1} \leqq 0\right) \\
& \geqq \mu \alpha_{1}+(m-\mu) \alpha_{\mu+1} \\
& \geqq \sum_{i=1}^{\alpha} \alpha_{i}+\sum_{i=\mu+1}^{m} \alpha_{i}=0 .
\end{aligned}
$$


Hence all equality signs hold, and one sees easily that all $\alpha_{i}=0$. To obtain (b), given $i, j$, note first that there exist $i_{1}, \cdots, i_{n}$ such that $D_{i, i_{1}}, \cdots, i_{n}(P) \neq 0$, and $D_{j, i_{1}, \cdots, i_{n}}(P) \neq 0$ which follows easily using only that $<m / 2$ points $P_{i}$ lie in any one hyperplane. Then set:

$$
\Pi^{(i, j)}=D_{i, i_{1}}, \cdots, i_{n} \cdot\left(D_{j, i_{1}}, \cdots, i_{n}\right)^{N_{0}-1} \cdot \Pi^{\left(i_{1}\right)} \cdots \cdots \Pi^{\left(i_{n}\right)} \cdot \Pi^{(j)} \text {. Q.E.D. }
$$

2. Theorem. There exist a quasi-projective scheme $M$, and a morphism $\psi:\left(\mathbf{P}^{n}\right)_{0}^{m} \rightarrow M$ such that $\left(\mathbf{P}^{n}\right)_{0}^{m}$ is a principal fibre bundle over $M$, with group PGL( $n)$.

Proof. Let

$$
\begin{array}{r}
X_{i_{1}}, \cdots, i_{m}=X_{i_{1}}^{(1)} \cdot X_{i_{2}}^{(2)} \cdots X_{i_{m}}^{(m)}, \quad S=Z\left[\cdots, X_{j}^{(i)}, \cdots\right], \\
R=Z\left[\cdots, X_{i_{1}}, \cdots, i_{m}, \cdots\right], \quad S_{0}=Z\left[\cdots, D_{i_{0}}, \cdots, i_{n}, \cdots\right],
\end{array}
$$

and

$$
R_{0}=S_{0} \cap R .
$$

The first step is:

(A) $R_{0}$ is finitely generated. First notice that $R_{0}$ is generated by the monomials in $D_{i_{0}}, \cdots, i_{n}$ 's that are homogeneous of the same degree in each set of variables $X_{*}^{(i)}$. Following Hilbert [1], use:

Lemma of Gordan. A finite system of homogeneous linear Diophantine equations has a finite set of positive integral solutions so that every other positive integral solution is a positive integral combination of them.

If the $D_{i_{0}, \cdots, i_{n}}$ are listed as $D^{(1)}, D^{(2)}, \cdots, D^{(N)}$, then the monomials $D^{(1)^{r_{1}}} \cdot D^{(2)^{r_{2}}} \cdots D^{(N)^{r_{N}}}$ in $R_{0}$ are those satisfying:

$$
\sum_{\substack{\left[1 \text { occurs among } \\ \text { subscripts of } D^{(i)}\right]}} r_{i}=\sum_{\substack{\left[2 \text { occurs among } \\ \text { subscripts of } D^{(i)}\right]}} r_{i}=\text { etc. }
$$

and the lemma applies. Q.E.D.

Now recall the well-known lemma [2]:

Lemma. $R=\sum_{n} R_{n}$ a graded ring, finitely generated over $R_{0}$. There exists an $N$ such that if $R(N)=\sum_{n} R_{N n}$, then $R(N)$ is finitely generated over $R_{0}$ by elements of $R(N)_{1}=R_{N}$.

Pick such an $N$ for $R_{0}$ and consider the inclusion $R_{0}(N) \subset R(N)$. This corresponds to a rational map of projective schemes:

$$
\Psi:\left(\mathbf{P}^{n}\right)^{m} \rightarrow \bar{M} .
$$

(B) $\Psi$ is defined on $\left(\mathbf{P}^{n}\right)_{0}^{m}$. By the usual translation into algebra, this means that if $P$ is a geometric point in $\left(\mathbf{P}^{n}\right)_{0}^{m}$, there is an $\alpha \in R_{0, N k}$, 
some positive $k$, such that $\alpha(P) \neq 0$. But by part (a) of the proposition, there is indeed a monomial $\pi \in R_{0, k}, \pi(P) \neq 0$. Q.E.D. Let $\psi=\bar{\psi} \mid\left(\mathbf{P}^{n}\right)_{0}^{m}$. It is immediate that, if $\pi_{i}: \operatorname{PGL}(n) \times\left(\mathbf{P}^{n}\right)_{0}^{m} \rightarrow\left(\mathbf{P}^{n}\right)_{0}^{m}$ are the morphisms of the action of the group $\operatorname{PGL}(n)$ and the projection, for $i=1,2$ resp., then $\psi \circ \pi_{1}=\psi \circ \pi_{2}$. We wish to show that given any geometric point $P \in\left(\mathbf{P}^{n}\right)_{0}^{m}$, there is an open sub-scheme $U \subset \bar{M}$, $\psi(P) \in U$, and a morphism $s: U \rightarrow\left(\mathbf{P}^{n}\right)_{0}^{m}$ such that

(i) $\psi \circ s=$ identity,

(ii) $U \times \operatorname{PGL}(n) \rightarrow^{A} \psi^{-1}(U)$ given by $(x, \sigma) \rightarrow \pi_{1}(\sigma, s(x))$ on geometric points, is an isomorphism.

When this is shown, it follows that $M=\psi\left(\left(\mathbf{P}^{n}\right)_{0}^{m}\right)$ is open in $\bar{M}$ and the theorem is fully proven. $s$ will be constructed by the help of a very simple "Typische Darstellung" (as such identities are called in classical invariant theory). Namely, seek

(i) $I_{i, j} \in R_{0, N k}, 0 \leqq i \leqq n$, and $1 \leqq j \leqq m$,

(ii) $\alpha_{j} \in S_{0}, 1 \leqq j \leqq m$, homogeneous in $X_{*}^{(\boldsymbol{k})}$, all $k$,

(iii) $\sigma_{i, j} \in S, 0 \leqq i, j \leqq n$, homogeneous in $X_{*}^{(k)}$, all $k$ with degree independent of $i$ and $j$, but dependent on $k$, such that

$$
\begin{array}{lrl}
\alpha_{j} X_{i}^{(j)}=\sum_{k=0}^{n} \sigma_{i, k} I_{k, j}, & 0 \leqq i \leqq n, 1 \leqq j \leqq m, \\
\alpha_{j}(P) & \neq 0, & 1 \leqq j \leqq m .
\end{array}
$$

Then $\left\{\sigma_{i, j}\right\}$ define a rational map $\phi:\left(\mathbf{P}^{n}\right)^{m} \rightarrow \operatorname{PGL}(n)$, while for each $j,\left\{I_{i, j}\right\}$ define a rational map $\bar{M} \rightarrow \mathbf{P}^{n}$, hence together a rational map $\bar{M} \rightarrow{ }^{S}\left(\mathbf{P}^{n}\right)^{m}$. Define $U \subset \bar{M}$ as the "set of points" $Q \in \bar{M}$ such that for all $j, \alpha_{j}(Q) \neq 0$. Clearly by $\left({ }^{*}\right), s$ is a morphism when restricted to $U$, and $\phi$ is a morphism when restricted to $\psi^{-1}(U)$. Moreover $\left(^{*}\right)$ then translates to the statement that the composed morphism below is the identity:

$$
\psi^{-1}(u) \stackrel{\phi \times(s \circ \psi)}{\longrightarrow} \mathrm{PGL}(n) \times\left(\mathbf{P}^{n}\right)^{m} \stackrel{\pi_{1}}{\rightarrow}\left(\mathbf{P}^{n}\right)^{m} .
$$

Then (i) follows formally, and to show (ii), define $B: \psi^{-1}(U) \rightarrow U$ $\times \operatorname{PGL}(n)$ by $\psi \times \phi$, then $A \circ B=$ identity also follows formally. To see that $B \circ A=$ identity, note first that for any geometric points $P$ and $\sigma$ in $\left(\mathbf{P}^{n}\right)_{0}^{m}$ and $\operatorname{PGL}(n)$ resp., $\pi_{1}(\sigma, P)=P$ implies $\sigma=e$; for there are clearly $n+1$ independent points $P_{i}^{\prime}$ among $P=\left(P_{1}, \cdots, P_{m}\right)$, and since $<m /(n+1)$ of the $P_{i}$ are in any one hyperplane, all $P_{i}$ could not lie in one of the $n+1$ hyperplanes spanned by $\left(P_{0}^{\prime}, \cdots, \hat{P}_{i}^{\prime}, \cdots, P_{n}^{\prime}\right)$. Consequently $B \circ A$ is the identity on geometric points, and since $U$ and $\operatorname{PGL}(n)$ are reduced, $B \circ A$ is the identity. 
Finally, construct the Typische Darstellung as follows. Given $P$, obviously there exist $i_{0}, \cdots, i_{n}$ such that $D_{i_{0}}, \cdots, i_{n}(P) \neq 0$. Simply multiply through by a suitable function the standard identity:

$$
D_{i_{0}, \cdots, i_{n}} X_{i}^{(j)}=\sum_{k=0}^{n} D_{i_{0}}, \cdots, \hat{i}_{k}, j, \cdots, i_{n} \cdot X_{i}^{\left(i_{k}\right)} .
$$

For some arbitrary $\alpha$, set

$$
\begin{aligned}
\alpha_{j} & =\left(D_{i_{0}}, \cdots, i_{n}\right)^{N_{0}} \cdot \Pi^{(\alpha, j)} \cdot \Pi^{\left(i_{0}, \alpha\right)} \cdots \cdot \Pi^{\left(i_{n}, \alpha\right)} \cdot \Pi^{\left(i_{0}\right)} \cdots \cdots \cdot \Pi^{\left(i_{n}\right)}, \\
I_{k, j} & =\left(D_{i_{0}, \cdots, i_{n}}\right)^{N_{0}-1} \cdot D_{i_{0}, \cdots, \hat{i}_{k}, j, \cdots, i_{n}} \cdot \Pi^{(\alpha, j)} \cdot \Pi^{\left(i_{k}, \alpha\right)} \cdot \Pi^{\left(i_{0}\right)} \cdots \cdot \Pi^{\left(i_{n}\right)}, \\
\sigma_{i, k} & =\Pi^{\left(i_{0}, \alpha\right)} \cdots \cdot \hat{\Pi}^{\left(i_{k}, \alpha\right)} \cdots \cdots \Pi^{\left(i_{n}, \alpha\right)} \cdot X_{i}^{\left(i_{k}\right)}
\end{aligned}
$$

and $\left({ }^{*}\right)$ and $\left({ }^{* *}\right)$ follow. Q.E.D.

\section{REFERENCES}

1. D. Hilbert, Über die Endlichkeit des Invariantensystems für binäre Grundformen, Collected Works, vol. 2, Berlin, 1933.

2. D. Mumford, Orbit spaces of varieties by linear groups in char. $O$, forthcoming.

3. A. Grothendieck, Théorie de descente. IV, Séminaire Bourbaki, mai, 1961.

HARVARD UNIVERSITY 